\title{
Wheels within Wheels: Hamiltonian Dynamics as a Hierarchy of Action Variables
}

\author{
Rory J. Perkins and Paul M. Bellan \\ Applied Physics, Caltech, Pasadena, California 91125, USA
}

(Received 28 April 2010; published 14 September 2010)

\begin{abstract}
In systems where one coordinate undergoes periodic oscillation, the net displacement in any other coordinate over a single period is shown to be given by differentiation of the action integral associated with the oscillating coordinate. This result is then used to demonstrate that the action integral acts as a Hamiltonian for slow coordinates providing time is scaled to the "tick time" of the oscillating coordinate. Numerous examples, including charged particle drifts and relativistic motion, are supplied to illustrate the varied application of these results.
\end{abstract}

DOI: 10.1103/PhysRevLett.105.124301

PACS numbers: 45.20.Jj, 45.50.Dd, 52.20.Dq

Hamiltonian dynamics is almost ubiquitous in physics and describes such varied phenomena as celestial mechanics, optics, fluid dynamics, quantum mechanics, and charged particle motion in electromagnetic fields. Guiding center theory, an approximation of Hamiltonian dynamics for charged particle motion in magnetic fields, describes the motion of the particle's cyclotron-orbit averaged position, or guiding center [1]. The guiding center can be thought of as a "quasiparticle" subject to new types of forces and manifesting various drifts. We develop a general model, not restricted to charged particle motion, of multidimensional systems with a periodic variable and find drifts that cannot be calculated using guiding center theory which becomes a limited example of the more general model. The model shows that the action integral associated with the oscillatory coordinate acts as an effective Hamiltonian for the remaining, slow coordinates providing time is measured in clock cycles of the oscillations. We note that Hamiltonian-type aspects of action integrals have been previously discussed in specific situations [2] but without developing a general demonstration and relying on the detailed equations of motion in their proofs. The model presented here generates a hierarchy of "wheelswithin-wheels" Hamiltonian systems such that the action integral associated with periodic motion at any level in the hierarchy acts as the Hamiltonian for the next slower periodic motion.

Consider a two-dimensional time-independent Hamiltonian system $H\left(\xi, P_{\xi}, P_{\eta}\right)$ with an ignorable coordinate $\eta$ and where the $\xi$ motion is periodic, i.e., $\xi(t+$ $\Delta t)=\xi(t)$, with no limit on the amplitude of $\xi . P_{\eta}$ evolves trivially: $\dot{P}_{\eta}=0$, but the $\eta$ evolution is in general nontrivial. The period $\Delta t$ can be imagined as a clock tick over which $\eta$ undergoes a net change $\Delta \eta$. We claim that

$$
\Delta \eta=-\partial J / \partial P_{\eta},
$$

where

$$
J\left(H, P_{\eta}\right)=\oint P_{\xi}\left(H, \xi, P_{\eta}\right) d \xi
$$

is the action integral (p. 454 in [3]) associated with $\xi$. Equation (1) means that if $J\left(H, P_{\eta}\right)$ is known, the net change of $\eta$ during one period of $\xi$ can be calculated without having to consider the potentially complicated form of $\dot{\eta}$.

To prove Eq. (1), first note that there is no contribution from differentiating the integral's bounds, so

$$
\frac{\partial J}{\partial P_{\eta}}=\oint \frac{\partial P_{\xi}}{\partial P_{\eta}} d \xi
$$

The differential of $H$ is

$$
d H=\frac{\partial H}{\partial \xi} d \xi+\frac{\partial H}{\partial P_{\xi}} d P_{\xi}+\frac{\partial H}{\partial P_{\eta}} d P_{\eta},
$$

so

$$
\frac{\partial P_{\xi}}{\partial P_{\eta}}=-\frac{\partial H / \partial P_{\eta}}{\partial H / \partial P_{\xi}} .
$$

Using Eq. (5) and Hamilton's equations in Eq. (3) gives

$$
\frac{\partial J}{\partial P_{\eta}}=-\oint \frac{\partial H / \partial P_{\eta}}{\partial H / \partial P_{\xi}} d \xi=-\oint \frac{d \eta / d t}{d \xi / d t} d \xi=-\Delta \eta .
$$

If there are other ignorable coordinates in the system, then suitably adjusted versions of Eq. (6) apply to each of them. Equation (1) generalizes the well-known theorem (p. 455 in[3]) that the period of motion is given by a partial derivative of $J$ with respect to $H$, namely,

$$
\Delta t=\partial J / \partial H \text {. }
$$

Equation (7) resembles Eq. (1) because $(t,-H)$ form a pair of canonical coordinates in extended phase space, so Eq. (7) is a special case of the theorem presented here. The drift, or net time evolution, of $\eta$ is clearly

$$
\frac{\Delta \eta}{\Delta t}=-\frac{\partial J / \partial P_{\eta}}{\partial J / \partial H}
$$

which generalizes the particle drifts associated with guiding center theory.

We now relax the requirement that $\eta$ is ignorable and allow the oscillations to evolve adiabatically. We do so by 
coupling the original Hamiltonian, now denoted as $H_{\mathrm{loc}}$, to an external system $H_{\text {ext }}$ that is otherwise isolated. This gives a total Hamiltonian

$$
H\left(\xi, P_{\xi}, \eta, P_{\eta}\right)=H_{\mathrm{loc}}\left(\xi, P_{\xi}, \eta, P_{\eta}\right)+H_{\mathrm{ext}}\left(\eta, P_{\eta}\right) .
$$

We presume the system behaves as follows. First, the $\xi$ oscillation is described by the local Hamiltonian $H_{\mathrm{loc}}$ in which $\eta$ and $P_{\eta}$ play the role of slowly varying parameters: $d \xi / d t=\partial H_{\mathrm{loc}} / \partial P_{\xi}$ and $d P_{\xi} / d t=-\partial H_{\mathrm{loc}} / \partial \xi$. Second, the "parametric" coordinates $\eta$ and $P_{\eta}$ are described by the total Hamiltonian $H$, so $d \eta / d t=\partial H / \partial P_{\eta}$ and $d P_{\eta} / d t=-\partial H / \partial \eta$. The local and external systems exchange energy, but the total energy $E=E_{\mathrm{loc}}(t)+E_{\mathrm{ext}}(t)$ is conserved since the entire system is isolated. $J$ is defined as in Eq. (2) but is now also a function of $\eta$. As in Ref. [4], we assume it is a good approximation to hold the parametric coordinates $\eta$ and $P_{\eta}$ fixed while evaluating the $\xi$ action integral. $J$ is an adiabatic invariant and is thus conserved. Furthermore, $J$ depends only on $H_{\text {loc }}$, i.e. $J=$ $J\left(H_{\mathrm{loc}}, \eta, P_{\eta}\right)=J\left(H-H_{\mathrm{ext}}\left(\eta, P_{\eta}\right), \eta, P_{\eta}\right)$, because $H_{\mathrm{loc}}$ is sufficient to prescribe the $\xi$ dynamics. A proof analogous to that of Eq. (1) shows

$$
\frac{\partial J}{\partial \eta}=\Delta P_{\eta}, \quad \frac{\partial J}{\partial P_{\eta}}=-\Delta \eta .
$$

Note that $J=J\left(H-H_{\text {ext }}\left(\eta, P_{\eta}\right), \eta, P_{\eta}\right)$ depends on $\eta$ and $P_{\eta}$ both implicitly through $H_{\text {ext }}$ and also explicitly. Accordingly, $\Delta \eta$ and $\Delta P_{\eta}$ have two terms: one term comes from the explicit dependence and is the drift of the system; the second term comes from the implicit dependence and is the slow change of $H_{\text {ext }}$.

Eqs. (10) have the makings of a Hamiltonian system with $-J$ serving as the Hamiltonian. They are precisely Hamiltonian as follows. We define discretized derivatives $d \eta / d t=\Delta \eta / \Delta t$ and $d P_{\eta} / d t=\Delta P_{\eta} / \Delta t$ that capture the net rates of change of $\eta$ and $P_{\eta}$. Upon invocation of a rescaled time $\tau$ normalized by the $\xi$ period:

$$
d \tau=d t / \Delta t
$$

Equations (10) become

$$
\frac{d \eta}{d \tau}=\frac{\partial}{\partial P_{\eta}}(-J), \quad \frac{d P_{\eta}}{d \tau}=-\frac{\partial}{\partial \eta}(-J) .
$$

Thus, $-J$ is the Hamiltonian for the averaged system provided time is measured in units of $\Delta t$. It should be noted that $\tau$ is the angle variable conjugate to $J$.

Alternatively, we can obtain a Hamiltonian for the $\xi$-averaged system by solving $J=J\left(H_{\mathrm{loc}}, \eta, P_{\eta}\right)$ for $H_{\mathrm{loc}}=H_{\mathrm{loc}}\left(J, \eta, P_{\eta}\right)$ which upon inserting in Eq. (9) gives

$$
H=H_{\mathrm{loc}}\left(J, \eta, P_{\eta}\right)+H_{\mathrm{ext}}\left(\eta, P_{\eta}\right) .
$$

Solution of Eq. (13) for $J$ gives $J=J\left(H, \eta, P_{\eta}\right)$. The differential of $J$ using this latter form is

$$
d J=\frac{\partial J}{\partial H} d H+\frac{\partial J}{\partial \eta} d \eta+\frac{\partial J}{\partial P_{\eta}} d P_{\eta}
$$

Equation (14) determines $\partial H / \partial P_{\eta}=-\left(\partial J / \partial P_{\eta}\right) /$ $(\partial J / \partial H)$, etc., so using Eqs. (10)

$$
\begin{aligned}
& \frac{\partial H}{\partial P_{\eta}}=-\frac{\partial J / \partial P_{\eta}}{\partial J / \partial H}=-\frac{-\Delta \eta}{\Delta t}=\frac{d \eta}{d t}, \\
& \frac{\partial H}{\partial \eta}=-\frac{\partial J / \partial \eta}{\partial J / \partial H}=-\frac{\Delta P_{\eta}}{\Delta t}=-\frac{d P_{\eta}}{d t} .
\end{aligned}
$$

Thus, $H$, written as Eq. (13), generates the discretized derivatives. The term $H_{\mathrm{loc}}\left(J, \eta, P_{\eta}\right)$ is an adiabatic potential [5], the residue of averaging the periodic $\xi$-motion. For systems approximating a harmonic oscillator, $J=$ $2 \pi H_{\mathrm{loc}} / \omega\left(\eta, P_{\eta}\right)$, so the adiabatic potential is $H_{\mathrm{loc}}=$ $J \omega / 2 \pi$, showing that $J$ acts like an electrostatic charge and $\omega\left(\eta, P_{\eta}\right)$ acts like an electrostatic potential. The magnitude of this " $J$ charge" depends on the amplitude of the $\xi$ oscillation. The use of $-J\left(H, \eta, P_{\eta}\right)$ as a Hamiltonian with normalized time $\tau$ and the use of $H\left(J, \eta, P_{\eta}\right)$ with regular time are entirely equivalent. Practically, though, there are techniques to evaluate $J$ directly [6], so using $-J$ as the Hamiltonian spares one from inverting $J$ for $H$, which might not be analytically feasible.

We now provide examples illustrating applications. Figure 1 shows an electron moving in the $r z$ plane and subjected to the magnetic field of a current-carrying wire aligned along the $z$ axis. The $z$ coordinate corresponds to $\eta$ and is ignorable; the radial motion is periodic and not ignorable because of the magnetic field gradient. The electron displaces itself an axial distance $\Delta z$ with every gyration as shown in Fig. 1. Using the characteristic velocity $\beta=\mu_{o} I e / 2 \pi m$ [7], the Hamiltonian is

$$
H=\frac{1}{2} m v^{2}=\frac{P_{r}^{2}}{2 m}+\frac{\left(P_{z}-m \beta \ln (r / R)\right)^{2}}{2 m},
$$

where $R$ is an arbitrary constant of integration. $J$ can be evaluated exactly [8] using the substitution $\cos \theta=\left(P_{z}-\right.$ $m \beta \ln (r / R)) / m v$ and the integral representation of the

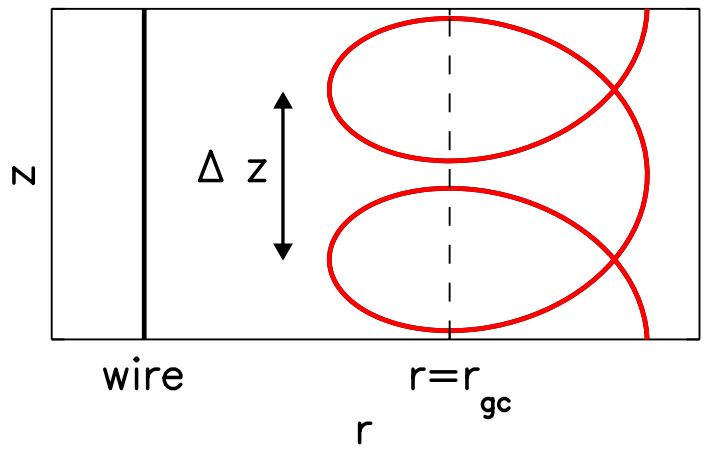

FIG. 1 (color online). For planar electron motion outside a current-carrying wire, the axial displacement $\Delta z$ can be derived from the radial action variable. 
modified Bessel function $I_{n}(x)=\pi^{-1} \int_{0}^{\pi} e^{x \cos \theta} \cos (n \theta) d \theta$ [9] so that

$$
J=\oint P_{r} d r=2 \pi m v r_{\mathrm{gc}} I_{1}\left(\frac{v}{\beta}\right)
$$

where $r_{\mathrm{gc}}=R \exp \left(P_{z} / m \beta\right) . r_{\mathrm{gc}}$, plotted as a dashed line in Fig. 1 , is the radial position at which the $z$ velocity vanishes, as can be checked from $P_{z}=m v_{z}+m \beta \ln r / R$. $J$ generalizes the first adiabatic invariant $\mu=m v_{\perp}^{2} / 2 B$ [1] and reduces to $2 \pi(m / e) \mu$ when $v \ll \beta$, which for this system is the condition for the guiding center approximation to hold [7]. $\Delta z$ and $\Delta t$ can be computed using Eqs. (1) and (7) and noting that $v=\sqrt{2 H / m}$. The exact drift velocity, computed without appealing to the guiding center approximation, is

$$
\mathbf{v}_{d}=\frac{\Delta z}{\Delta t} \hat{z}=-v \frac{I_{1}(v / \beta)}{I_{0}(v / \beta)} \hat{z} .
$$

Equation (19) holds for orbits of all energies even when the guiding center approximation fails. The $v \ll \beta$ limit of Eq. (19) reduces to the $\operatorname{grad} B$ drift [10] of the guiding center approximation.

Next we show how $J$ can be used as a Hamiltonian to give the magnetic mirror force [10]. For a magnetic field mainly in the $z$ direction, the cyclotron motion is essentially harmonic oscillation at the gyrofrequency $\omega=$ $q B / m$ in the perpendicular direction, so we identify $\eta$ with $z$ and $H_{\text {ext }}$ with $P_{z}^{2} / 2 m$. Then

$$
J=2 \pi \frac{H_{\mathrm{loc}}}{\omega}=2 \pi \frac{H-P_{z}^{2} / 2 m}{q B / m}=2 \pi \frac{m v_{\perp}^{2} / 2}{q B / m},
$$

which equals $\mu$ except for the factor $2 \pi m / q$. If $B$ depends on $z$ then Eqs. (15) and (16) become

$$
\begin{array}{r}
\frac{d z}{d t}=-\frac{\partial J / \partial P_{z}}{\partial J / \partial H}=\frac{P_{z} / m}{q B / m} \frac{q B}{m}=\frac{P_{z}}{m}, \\
\frac{d P_{z}}{d t}=\frac{\partial J / \partial z}{\partial J / \partial H}=-\frac{q J}{2 \pi m} \frac{\partial B}{\partial z}=-\mu \frac{\partial B}{\partial z},
\end{array}
$$

establishing the magnetic mirror force without considering the microscopic motion.

A slightly different approach retrieves the $\operatorname{grad} B$ drift. Suppose $\mathbf{B}=B_{z}(x) \hat{z}$, so $\mathbf{A}=A_{y}(x) \hat{y}$ with $B_{z}=\partial A_{y} / \partial x$. We define the $x$ component of the guiding center as the position $x_{\mathrm{gc}}$ where $v_{y}$ vanishes: $P_{y}=q A_{y}\left(x_{\mathrm{gc}}\right)$. Setting $\eta=y$, Eq. (15) applied to Eq. (20) gives

$$
\frac{d y}{d t}=-\frac{\partial J / \partial P_{y}}{\partial J / \partial H}=\mu\left(\frac{\partial B_{z}}{\partial x}\right)_{x_{\mathrm{gc}}} \frac{\partial x_{\mathrm{gc}}}{\partial P_{y}} .
$$

We then use $\partial x_{\mathrm{gc}} / \partial P_{y}=\left(q B_{z}\left(x_{\mathrm{gc}}\right)\right)^{-1}$, obtained by differentiating $P_{y}=q A_{y}\left(x_{\mathrm{gc}}\right)$ with respect to $P_{y}$. Equation (23) thus becomes

$$
\frac{d y}{d t}=\frac{\mu}{q B_{z}}\left(\frac{\partial B_{z}}{\partial x}\right)_{x_{\mathrm{gc}}}
$$

which is the $\operatorname{grad} B$ drift.

A surprising application arises in relativistic mechanics, where it is found that in crossed electric and magnetic fields $\mathbf{E}=E \hat{x}$ and $\mathbf{B}=B \hat{z}$ with $E<B c$ a particle's $z$ velocity is modulated by the cyclotron motion, in contrast to the nonrelativistic situation where $v_{z}$ is constant and independent of the cyclotron motion. The modulation arises from the periodic addition and subtraction of the $\mathbf{E} \times \mathbf{B}$ drift to the cyclotron velocity, which modulates $\gamma=\left(1-v^{2} / c^{2}\right)^{-1 / 2}$ and hence the particle's effective mass; $v_{z}$ then varies because $v_{z}=P_{z} / \gamma m$ and $P_{z}$ is invariant as $z$ is ignorable. Using the relativistic canonical momenta $\mathbf{P}=m \gamma \mathbf{v}+q \mathbf{A}$ with $\mathbf{A}=B x \hat{y}$, the electrostatic potential $\phi=-E x$, and the relativistic Hamiltonian $H=$ $c \sqrt{(\mathbf{P}-q \mathbf{A})^{2}+m^{2} c^{2}}+q \phi$, it is found that the relativistic $x$ action $J=\oint P_{x} d x$ evaluates to

$$
\frac{J}{2 \pi}=\frac{\left(B P_{y}+H E / c^{2}\right)^{2}}{2 q\left(B^{2}-E^{2} / c^{2}\right)^{3 / 2}}+\frac{\left(H^{2} / c^{2}-P_{z}^{2}-P_{y}^{2}-m^{2} c^{2}\right)}{2 q\left(B^{2}-E^{2} / c^{2}\right)^{1 / 2}} .
$$

Calculating $\Delta z$ and $\Delta t$ using Eqs. (1) and (7) gives the $E$-dependent $z$-drift velocity

$$
v_{d}=\frac{\Delta z}{\Delta t}=\frac{B^{2}-E^{2} / c^{2}}{B E P_{y} / c^{2}+B^{2} H / c^{2}} P_{z} .
$$

As shown in Fig. 2, this $v_{z}$ drift has been verified by direct numerical integration of the relativistic equation of motion $d(\gamma m \mathbf{v}) / d t=q(\mathbf{E}+\mathbf{v} \times \mathbf{B})$ which shows that the modulation of $v_{z}$ is typically spiky as $\gamma \approx 1$ for a short interval during each cyclotron period and then $\gamma \gg 1$ for the remaining fraction of the cyclotron period. Clearly, this

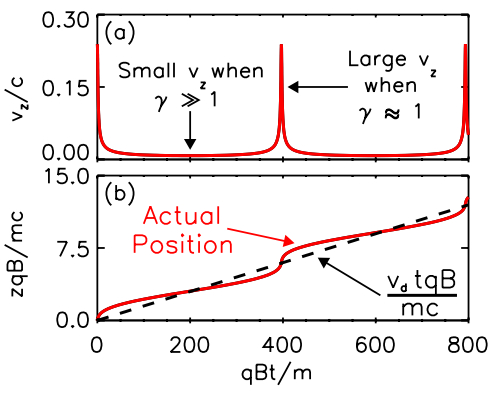

FIG. 2 (color online). A particle undergoing relativistic $E \times B$ motion in the $x y$ plane with $E=0.95 B c$ and initial momentum $P_{x}=0, P_{y}=0.7 \mathrm{mc}$, and $P_{z}=0.3 \mathrm{mc}$. (a) The $z$ velocity (from numerical integration of the relativistic equation of motion) is nonconstant, spiking when the particle's $x y$ motion slows down so that $\gamma \approx 1$. (b) The solid line is the numerically integrated $z$ position; dashed line, calculated using Eq. (26), captures the $z$-drift motion. 
analysis generalizes to force-drifts by replacing $\mathbf{E}$ with $\mathbf{F} / q$.

Kepler motion provides a nonrelativistic and nonelectromagnetic example. The radial action is (p. 469 in [3])

$$
J_{r}=-2 \pi\left|P_{\phi}\right|+2 \pi m^{3 / 2} M G / \sqrt{2|H|},
$$

where $P_{\phi}$ is the conserved angular momentum. Equation (1) gives $\Delta \phi= \pm 2 \pi$ depending on the sign of $P_{\phi}$, immediately proving that bounded Kepler orbits are always closed.

We now present a purely mechanical system which exhibits the equivalent of "magnetic" mirroring. Consider a nonrelativistic particle in a long groove where the width of the groove varies with position. The Hamiltonian is

$$
H=\frac{P_{x}^{2}}{2 m}+\frac{P_{y}^{2}}{2 m}+\frac{1}{2} \kappa x^{2}\left(1+\alpha y^{2}\right)+\frac{\lambda}{2} y^{2},
$$

where $y$ is the distance along the groove and $x$ is the distance across the groove. Presuming that the $y$ position changes slowly relative to the oscillations across the groove (i.e., $|\alpha|$ and are small compared to $\kappa$ ), the $y$-dependent frequency of $x$ oscillation is

$$
\omega(y)=\sqrt{\frac{\kappa}{m}} \sqrt{1+\alpha y^{2}} .
$$

We identify $H_{\text {loc }}=P_{x}^{2} / 2 m+m \omega(y)^{2} x^{2} / 2$, so the $x$ action is $J=2 \pi H_{\text {loc }} / \omega(y)$, and Eq. (13) becomes

$$
H=\frac{P_{y}^{2}}{2 m}+\frac{\lambda}{2} y^{2}+\frac{\omega(y)}{2 \pi} J
$$

Equation (16) gives an average force $-(J / 2 \pi) \partial \omega / \partial y=$ $-y J \kappa \alpha / 2 \pi m \omega$ in the $y$ direction due to the increase in $x$ oscillation energy where the groove narrows. This is a restoring force and, if sufficiently strong, can overwhelm the contribution from $\lambda$. A negative $\lambda$ corresponds to a potential hill, and if $J=0$ the particle falls down the hill. However, if $J$ is sufficiently large and $\alpha$ is positive, the particle does not fall down but instead oscillates about the top of the hill! This mechanical analog of a magnetic mirror has been verified by direct numerical integration as shown in Fig. 3.

For oscillatory y motion, Eq. (30) admits an action integral in the $y$ direction, which we denote by $K$, that acts as a Hamiltonian for the $x$-averaged system. This is a two-tier hierarchy of action variables, or a wheel within a wheel. For the reduced system, $J$ is a conserved quantity, so we develop an analog of Eq. (1):

$$
\begin{gathered}
\frac{\partial K}{\partial J}=\oint \frac{\partial P_{y}(H, J, y)}{\partial J} d y=\oint \frac{1}{\partial J / \partial P_{y}} d y \\
=\oint \frac{-1}{d y / d \tau} d y=-\Delta \tau
\end{gathered}
$$

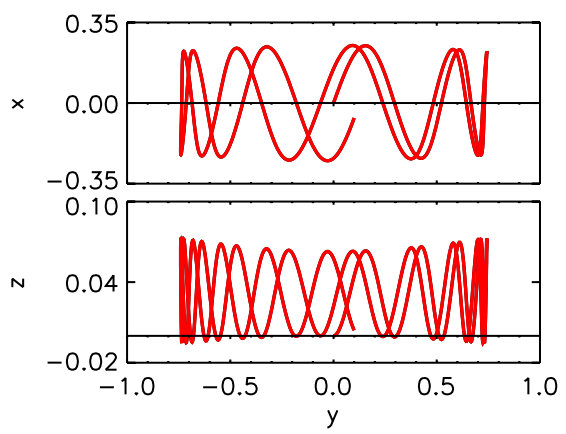

FIG. 3 (color online). A particle in a thin saddlelike groove can undergo oscillatory motion due to narrowing of the groove. $x$ is the direction across the groove, $y$ along the groove, and $z$ the vertical. $H$ is given by Eq. (28) with $m=\kappa=\alpha=1$ and $\lambda=$ -0.01 , and the particle starts at $x=y=0$ with $v_{x}=0.25$ and $v_{y}=1$.

where we use Eq. (14) to evaluate $\partial P_{y} / \partial J$ and Eq. (12) to evaluate $\partial J / \partial P_{y}$. Since $\tau$ counts $x$ cycles, $-\partial K / \partial J$ gives the number of $x$ cycles per $y$ cycle. If this quantity is a rational number, the trajectory is closed. This is of interest when quantizing the system, as there is sometimes a one-to-one correspondence between periodic classical trajectories and quantum energy levels [11].

Supported by U.S. DOE and NSF.

[1] H. Alfvèn, Cosmical Electrodynamics (Clarendon Press, Oxford, 1950), 1st ed.

[2] B. B. Kadomtsev, Plasma Physics and the Problem of Controlled Thermonuclear Reactions (Pergamon Press, New York, 1959), Vol. III, p. 340; T. G. Northrop and E. Teller, Phys. Rev. 117, 215 (1960); R. B. White and M. S. Chance, Phys. Fluids 27, 2455 (1984).

[3] H. Goldstein, C.P. Poole, and J.L. Safko, Classical Mechanics (Addison Wesley, San Francisco, 2002), 3rd ed.

[4] L. D. Landau and E. M. Lifshitz, Mechanics (Pergamon Press, New York, Oxford, 1969) p. 154.

[5] K. Stefanski and H. S. Taylor, Phys. Rev. A 31, 2810 (1985).

[6] M. Born, The Mechanics of the Atom (F. Ungar Pub. Co., New York, 1960) appendix II.

[7] J. Neuberger and J. Gruenebaum, Eur. J. Phys. 3, 22 (1982).

[8] M. J. Wouters, J. Phys. D 28, 1547 (1995).

[9] M. Abramowitz and I. Stegun, Handbook of Mathematical Functions (Dover, New York, 1970), Eq. 9.6.19.

[10] P. M. Bellan, Fundamentals of Plasma Physics (Cambridge University Press, Cambridge, England; New York, 2006) p. 96, 101.

[11] M. Gutzwiller, J. Math. Phys. (N.Y.) 11, 1791 (1970). 